\title{
Food Patterns
}

National Cancer Institute

\section{Source}

National Cancer Institute. Food Patterns. NCI Thesaurus. Code C16045.

The types of food combinations that are commonly eaten by a population. 